\title{
POURQUOI LE PRIX NOBEL DE LITTERATURE A-T-IL ESSUYE DES CRITIQUES?
}

\author{
Refika PARLAK ${ }^{1}$
}

\begin{abstract}
Öz: Nobel ödülleri de sinema ve müzik alanında verilen Oscar ve Grammy ödülleri gibi dünyanın en görkemli ödüllerinden olup her yıl kime ya da kimlere, dil, din, irk ayırt etmeksizin, verileceği dünya kamuoyu tarafindan merakla beklenir. Fizik, Kimya, Edebiyat, Tıp ve Ekonomi bilim dallarında ve de Barış alanında İsveç Kraliyet Bilimler Akademisi tarafından verilen bu ödüllerin kime, neden, nasıl değer görüldüğü tartışma konusu olduğu gibi, ödüle değer adaylar da gündemi uzun süre meşgul eder. Dinamitin icatçısı olarak bilinen ve bu anlamda insanlığa karşı büyük bir suç işlediği varsayılan Alfred Nobel (18331896)'in servetinin insanlığa katkısı olanlara ödül olarak dağıtılmasını vasiyet etmesi Nobel ödüllerinin tartışılmasına neden olmuştur. Söz konusu ödüllerle ilgili her daim güncelliğini koruyan bir diğer tartışma ise bu ödüller verilirken gerçekten adayların seçimi nesnel yapılabiliyor mu ya da önceden birilerine söz mü veriliyor? Ve İsveç kraliyetinin dağıttığ1 bu ödüller ne denli güvenilirdir? $\mathrm{Bu}$ çalışmada akıllara takılan bu ve benzeri soruları (Nobel Edebiyat ödülü en çok hangi ülkeye verilmiştir? İsveç akademisi dünya dilleri arasında olmayan "azınlık dillere" ne denli şans tanıyor? Bu saygın ödülün sahibi olmak için yazarların "taraflı" olması şart mıdır? Kadın yazarların erkek yazarlara kıyasla şansları var mıdır?) diğer bütün Nobel ödüllerinde olduğu gibi, tarih boyunca hikâyesi ile tartışmaların odağında yer alan Nobel Edebiyat ödülü çerçevesinde yanıtlamaya çalışacağız.
\end{abstract}

Anahtar Sözcükler: Nobel Edebiyat Ödülü, Siyaset, İsveç Akademisi, Yazar, Eserler.

\section{Introduction}

Comme nous le savons, le $\mathrm{XXI}^{{ }^{\mathrm{ème}}}$ siècle se développe sous le signe de la mondialisation qui trouve son origine dans les échanges économiques (idée de marché sans frontière, pas de nation universelle bien que l'uniformisation culturelle participerait à une uniformisation du profil des consommateurs, sous certaines limites). En ce sens le dialogue interculturel devient inévitable, ainsi occupe une place primordiale en ne pouvant se réaliser qu'en prenant conscience de son propre « moi » à travers «l'autre». En revanche l'explosion

1 Doktora Öğrencisi, Uludağ Üniversitesi, Eğitim Bilimleri Enstitüsü, Fransız Dili Eğitimi Anabilim Dal..rfkprlk@hotmail.com 
des nouvelles technologies de l'information (Internet, mobiles...) a pour conséquence au $\mathrm{XXI}^{\mathrm{e} m e}$ d'amplifier considérablement le phénomène ce qui facilite cet échange. Nous allons surtout mettre en avant la littérature qui représente un moyen qui privilégie la communication entre les cultures, et qui établit donc un dialogue entre les expressions culturelles multiples. Ainsi comme le dit Sergio Kokis, célèbre romancier et peintre contemporaine, c'est la littérature qui explique le mieux l'être humain (Tétu de Labsade, 1997) et c'est pourquoi le choix de la langue d'écriture pour un écrivain est important s'il veut se faire comprendre de tous les lecteurs de toutes les cultures. Tout écrivain a alors bien intérêt d'écrire dans le but de séduire le grand public pour être lu, apprécié et compris de tous. La question qu'on se pose donc ça sera de savoir comment prendre conscience de tous les écrivains du monde entier et de leurs œuvres. Or, comme dans le domaine cinématographique, musicale etc., dans le domaine littéraire aussi des prix sont décernés aux meilleurs de l'année. Par conséquent, c'est dans ce sens qu'il va être intéressant d'interroger le rôle des prix littéraires.

Il existe un grand nombre d'institutions qui se chargent de couronner les auteurs ayant écrit des œuvres remarquables tel que le prix roman d'Orhan Kemal, le prix Goncourt, le prix Médicis, le prix Booker. Or on mettra l'accent sur le prix Nobel de la littérature, le plus prestigieux aux yeux de tous, afin de pouvoir répondre à toutes les questions. En effet, le prix littéraire international le plus renommé est celui qui est décerné par l'Académie suédoise à Stockholm. Son fonctionnement est inhabituel, sa nomination reste un mystère pour tous jusqu'au verdict. Bien que les journalistes fassent des sondages, pour tenter de deviner qui sera le lauréat du prix littéraire chaque année, il est difficile de le saisir. Cependant, le testament d'Alfred Nobel (1833 - 1896) peut-il en effet nous laisser entendre quelques indices?

Tout le reste de la fortune réalisable que je laisserai en mourant sera employé de la manière suivante : le capital placé en valeurs mobilières sûres par mes exécuteurs testamentaires constituera un fonds dont les revenus seront distribués chaque année à titre de récompense aux personnes qui, au cours de l'année écoulée, auront rendu à l'humanité les plus grands services. Ces revenus seront divisés en cinq parties égales. La première sera distribuée à l'auteur de la découverte ou de l'invention la plus importante dans le domaine de la physique; la seconde à l'auteur de la découverte ou de l'invention la plus importante en chimie; la troisième à l'auteur de la découverte la plus importante en physiologie ou en médecine; la quatrième à l'auteur de l'ouvrage littéraire le plus remarquable d'inspiration idéaliste; la cinquième à la personnalité qui aura le plus ou le mieux contribué au rapprochement des peuples, à la suppression ou à la réduction des armées permanentes, à la réunion ou à la propagation des congrès pacifistes. Les prix seront décernés : pour la physique et la chimie par l'Académie suédoise des Sciences, pour la physiologie ou la médecine par l'Institut Carolin de Stockholm, pour la littérature par l'Académie de Stockholm, et pour la défense de la paix par une commission de cinq membres élus par la "Storting" norvégienne. Je désire expressément que les prix soient décernés sans aucune considération de 
nationalité, de sorte qu'ils soient attribués aux plus dignes, scandinaves ou non ${ }^{2}$. (Nobel, 1895, p. 21).

Une chose est bien sûr, c'est que depuis que cette lourde tâche lui a été confiée, il s'est écrit une histoire jalonnée de controverses. Le domaine de la littérature se voit confronté à celui de la politique, puis certains prix se voient contestés, refusés, voire oubliés...

\section{Objectifs du travail}

Bien qu'il soit l'un des prix les plus prestigieux et controversés au niveau international, il n'existe pas de nombreuses recherches à ce sujet dans le monde, mis à part l'ouvrage d'Espmark, la référence absolue, intitulé Le prix Nobel, Histoire intérieure d'une consécration littéraire (1986) où l'auteur nous parle de la chronique du prix du début du siècle à nos jours. Il en est de même pour le cas de la Turquie où, hormis quelques articles concernant le prix Nobel de Orhan Pamuk (Gökalp, 2010 et Vural, 2007) la thèse de master de Ümran Küçüksüleymanoğlu (2014) dont l'objectif est de mettre en évidence l'importance de la traduction dans l'obtention du prix Nobel de Littérature qui se charge de nourrir la littérature mondiale et l'article de Alkan Soyak (2003) concernant le prix Nobel d'économie qui souligne la priorité que le comité accorde à ceux qui optent pour une approche orthodoxe en économie. Donc voici les questions autour desquelles notre texte va s'organiser:

1. Quels sont les critères d'éligibilité du comité Nobel?

2. L'honneur de remporter ce prix a-t-il été plus accordé aux pays occidentaux ou orientaux?

3. Pourquoi plus de lauréats que de lauréates?

4. Quel est le degré de chance d'un écrivain qui œuvre dans une langue qui lui est «inhabituelle» pour les membres du comité?

5. En dernier lieu, est-ce que le point de vue ou la position politique du lauréat est la clef de l'obtention du prix Nobel littéraire?

\section{Les prix Nobel}

Sur le modèle de l'Académie Française, le roi Gustave III souhaite fortifier l'expansion culturelle du royaume suédois et met en place, en 1786, 1'Académie Suédoise, qui est «une des académies royales de Suède» et dont la devise est «talent et goût» traduit du suédois snille och smak. Composé de dix-huit membres «issus du milieu des lettres et des langues», nommés à vie, tout comme l'Académie Française, elle a un rôle bien défini. Elle doit veiller sur «la pureté, la vigueur et la majesté» de la langue suédoise. Pour cela elle va faire paraître deux dictionnaires: a) Svenska Akademiens Ordlista (SAOL), en un seul volume; b) Svenska Akademiens Ordbok (SAOB), en plusieurs volumes concernant tous les mots de la langue.

\footnotetext{
${ }^{2}$ Disponible en ligne: http://www.nobelprize.org/alfred_nobel/will/will-full.html, consulté le 02 décembre 2014.
} 
Un seul dictionnaire sera finalement publié. Dès 1791, l'Académie Suédoise se voit confier la charge de rédaction du journal officiel Postoch Inrikes Tidningar. Cependant ce qui va lui donner la chance d'être une puissance mondiale, est enfoui dans le testament de l'inventeur de la dynamite, Alfred Nobel. Ce dernier né à Stockholm en 1833, fera fortune et s'illustrera à travers le monde grâce à son brevet d'invention de la dynamite. Un an avant sa mort, il se met à écrire son testament, et comme tout testateur nous fait part de ses héritiers en disant qu'il souhaite léguer sa fortune afin qu'une institution soit créée dans le but de rendre hommage aux personnes ayant apporté le plus grand bénéfice à l'humanité. Ces propos nous laissent entendre une certaine culpabilité de la part du «marchant de la mort» ${ }^{3}$ qui s'éteindra le 10 décembre 1896. Les revenus du capital seront attribués et à être partager sous forme de prix afin de couronner les hommes de bonnes volonté. Cinq prix annuel dans les domaines de la paix, de la médecine, de la chimie, de la physique et de la littérature seront distribués, auxquels vient s'ajouter en 1968 un prix en économie qui a été institué par la Banque de Suède, avec l'accord de la fondation Nobel.

La mission délicate d'accorder le prix Nobel de la paix est confiée au comité le Storting nommé par le parlement Norvégien qui siège à Oslo. L'institut universitaire médicale Carolin situé à Stockholm, qui représente l'un des centres de recherche médicale et est le groupement universitaire le plus important de l'Union Européenne, se chargera de décerner le prix Nobel de la médecine, l'Académie Royale des sciences (de Suède) celui de la chimie et de la physique et depuis 1968, comme on l'a déjà cité auparavant le prix Nobel d'économie sera décerné par la Banque de Suède en mémoire d'Alfred Nobel.

\section{Le prix Nobel de littérature}

Nombre de débats ont eu lieu au sein de cette dernière par crainte de subir une pression internationale et d'être écartée de sa réelle fonction qui était, comme on l'a dit précédemment, de veiller sur «la pureté, la vigueur et la majesté» de la langue suédoise. Un comité Nobel constitué de cinq personnalités choisies parmi les académiciens pour une durée de trois ans, mis en place afin d'examiner «la pertinence et le critère d'éligibilité des écrivains» dont le nom figure sur la liste des lauréats inconnus de tout le monde jusqu'au verdict. Chaque année avant le $1^{\text {er }}$ février, environ 350 noms sont proposés par des personnes habilitées dans leurs domaines, qui font tout autant partie de l'Académie en exerçant leur métier de professeur de littérature et de langue. Par la suite tous les jeudis qui suivront cette date jusqu'à mai, les membres du comité se réuniront à 17 heures afin de discuter les différentes œuvres et auteurs. Cependant beaucoup de candidatures se verront recalées à chaque réunion afin d'obtenir une liste secrète sur laquelle est inscrit le nom de cinq auteurs. De mai jusqu'à octobre les membres se verront à nouveau réunis dans le but d'étudier en détail l'œuvre des cinq finalistes. Pour conclure, le secrétaire perpétuel convoquera une dernière fois les membres de l'Académie le jeudi

\footnotetext{
${ }^{3}$ Titre qu'un journal français accorda à son article afin de publier la nécrologie d'Alfred Nobel
} 
suivant, qui correspond aux premiers jours d'octobre, à onze heures et demie pour procéder à un vote définitif. Après une heure et demie de délibération, le nom de l'auteur ayant obtenu plus de la moitié des voix sera désigné comme lauréat du prix. Gardé comme un secret absolu jusqu'à la dernière minute, l'auteur et l'œuvre digne d'un «puissant idéal» sont enfin rendus au grand public. Cependant les quatre candidats éliminés sont d'office réinscrits sur la liste de l'année suivante. Comme pour tout auteur d'une œuvre remarquable d'une «tendance idéaliste», la récompense sera décernée par le roi de Suède lors de la cérémonie qui aura lieu le 10 décembre, la date d'anniversaire de mort d'Alfred Nobel. Le lauréat remportera alors une médaille d'or, un document calligraphié témoignant son statut ainsi que les motifs pour lesquels il a été sélectionné parmi tant d'autres et son chèque de dix millions de couronnes (environ 1,1 million d'euros). Toutefois, un discours cérémonial, concernant son chef-d'œuvre et son «engagement artistique» sera attendu de sa part. Il est à noter que le contenu de ses réunions ainsi que le nom des cinq personnalités restera confidentiel pendant cinquante ans. Le prix Nobel de la littérature, lui sera décerné par l'Académie suédoise.

\section{Le prix Nobel de Littérature: Une histoire jalonnée de débats}

Après avoir pris connaissance de ce que représente le prix Nobel littéraire décerné par l'Académie suédoise à Stockholm, il est temps d'examiner pourquoi il est au centre de nombreuses spéculations. Tout d'abord, le prix ne va pas rendre plus célèbre certains auteurs déjà très connu du monde tel que Ernest Hemingway (1954), André Gide (1947) et bien d'autres encore. Certes, pour des auteurs moins réputés, c'est une chance à ne pas manquer pour être connu du grand public international, comme ce fut le cas pour le romancier Hongrois Imre Kertész (1929 -...) et l'écrivain Turc Orhan Pamuk $(1952$ - ...) Le prix a rendu, non seulement leurs ouvrages visibles mais leur a également attribué la valeur du «meilleur roman de l'année». Depuis 1901 l'Académie a désigné tous les ans un détenteur du prix Nobel de la littérature et suite à une analyse détaillée de la liste des lauréats (Tableau 1) il est remarquable que certains choix ont pu susciter des critiques. 
Tableau 1 : Liste des lauréats du prix Nobel littéraire

\begin{tabular}{|c|c|c|c|}
\hline 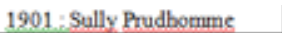 & 1930 : Sinclai & | 1959 : Salvatore Quasimodo & 1988: Naguib Mahfour \\
\hline 1902 Theodor Mommsen & 1931: E Axel Karlfeldt & 1960 - Saint-John Perse & 1989: Camilo José Cela \\
\hline 1903 -Bjomstieme Bjomson & 1932 : J. G. Drapeam & 1961 - Tro André & 1990: Octavio Paz \\
\hline 1904 . F et J. de Echegaray & 1933: Ivan Bourine & 1962: John Steinbeck & 1991: Nadine Gordimer \\
\hline 1905: Hennk Sienkiewicz & 1934: Luigi Pirandello & 1963: Georges Séferis & 1992: Derek Walcott \\
\hline 1906-Giosuè Carducci & 1935: non decemé & 1964-Jean-Paul Sartte & 1993: Toni Morriscon \\
\hline 1907 :Rudyard Kipling & 1936: Eugene ONcill & 1965 - Mikha1 Cholokhoy & 1994: Kenzaburo $0_{c}$ \\
\hline 1908 - R. Christoph Eucken & 1937 : R Martin du Gard & 1966 :S. Y. Agmon et N. Sachs & 1995: Seamus Heaney \\
\hline 1909-SeIma Lageriof & 1938 : Pearl Buck & 1967:M Angel Asturias & 1996:W Srymborska \\
\hline 1910: Paul Heyse & 1939 : F. Eemil Sillanpăa & 1968: Yasunari Kawabata & 1997: Dario Fo \\
\hline 1911- Maurice Maeterlinck & 1940: non décemé & 1969: Samuel Beckett & 1998: José Sarama80 \\
\hline 1912: Gethant Hauptmam & 1941: non decanc & 1970: Alexandre Soljenitsyne & 1999: Günter Grass \\
\hline 1913: Rabindranath Tagors & 1942: non-decems & 1971: Pablo Neruda & 2000: Gao Xingian \\
\hline 1914:non décemé & 1943 inon décemé & 1972: Heinnich Botl & 2001:V S. Naipaul \\
\hline 1915: Romain Rolland & 1944:J.V. Jensen & 1973 : Patrick Whitc & 2002: Imre Kertesz \\
\hline 1916 V Y yon Heidenstam & 1945: Gabricla Mistral & 1974 : E. Johnson et $H$ Martinsca & 2003:J.M. Coetzes \\
\hline 1917: K A Gjellenup & 1946: Hermann Hesse & 1975 -Eugenio Montale & 2004: Elfried Jelinek \\
\hline 1918 non dosane & 1947: Andre Gide & 1976 : Saul Bellow & 2005: Harold Pinter \\
\hline 1919: Carl Spitteler & 1948: T. S. Eliot & 1977 -Vicente Alcixandre & 2006: Othan Pamuk \\
\hline 1920 : K P. Hamein & 1949 : William Faulkner & 1978 - I B Singer & 2007: Doris Lessing \\
\hline 1921 Anatole France & 1950: B Arthur William & 1979 Odysscas Elytis & 2008: J. M. G. LeClezio \\
\hline 1922 - Jacinto Benavente & 1951 : Par Lagerkvist & 1980 - Creshaw Milosz & 2009 : Herta Mfuller \\
\hline 1923 -W, B Yeats & 1952: Franccis Mauriac & 1981 - Flias Canetti & 2011: Tomas Transtromes \\
\hline 1924 : W. S. Reymont & 1953 :W.L.S. Churchill & 1982: G. G. Marquez & 2012: Mo Yan \\
\hline $1925: G$ Bemard Shaw & 1954 : Emest Hemingway & 1983 - William Golding & 2013: Alice Munro \\
\hline 1926 - Grazia Deledda & 1955 - Halldor L amess & 1984 - Jaroslav Seifert & 2014 : Patrick Modiano \\
\hline 1927 : Henri Bergson & 1956: Juan Ramón Jiminez & 1985 Claude Simon & \\
\hline 1928 - Sigid Undset & 1957: Albert Camus & 1986: Wole Soyinka & \\
\hline 1929 Thomss Mann & 1958 : Boris Pastemak & 1987 Joscph Brodsky & \\
\hline
\end{tabular}

D'autant plus que ce prix littéraire, dont l'intérêt principal est de couronner «l'auteur de l'ouvrage littéraire le plus remarquable d'inspiration idéaliste», sera attribué assurément moins selon le critère d'«idéalisme» fixé par le testateur que par la perception et l'interprétation qu'en aurait de cette notion le secrétaire perpétuel, soulevant ainsi les spéculations (Epsmark, 1986, p. 166). Par ailleurs les deux guerres mondiales à leur tour vont jouer un rôle important dans le choix de certaines personnalités. Bien que la priorité soit accordée aux romanciers, poètes, dramaturges et essayistes, on comptera parmi les lauréats, les noms de Winston Churchill (historien), Christian Mattias Theodor (historiens), Rudolf Christoph Eucken (philosophe), Henri Bergson et Bertrand Russel (philosophe). Ces philosophes et historiens se distinguent soit par leurs discours politiques, soit par leurs philosophies.

La question se pose de savoir si des erreurs ont été commises lors de l'attribution des prix. La réponse paraît pourtant simple lorsqu'on pense aux «oubliés», «écartés», ou ceux qu'on considère «indigne» du prix. À ce sujet, dans son ouvrage, Espmark (1986) balaie les différents points de vue des membres de l'Académie afin de rendre compte à ses lecteurs des raisons pour lesquelles certains auteurs ont été préférés à d'autres, pourquoi certains ont été écartés ou oubliés. En ce qui concerne l'attribution du prix Nobel littéraire, Espmark dit qu'il faudrait faire une distinction entre les périodes où les prix Nobel littéraires ont été décernés. Les prix décernés au début du $\mathrm{XX}^{\mathrm{ème}}$ siècle, puis ceux attribués dans l'entre-deux Guerre (1914 - 1918 / 1939 - 1945), et enfin ceux distribués de nos jours n'ont pas la même valeur. C'est pendant l'ère du secrétaire permanent Wirsén (1884 - 1912) que Espmark (1986) a nommé 
«un idéalisme noble et sain», que l'on verra des écrivains tel que Tolstoï, Zola, Strindberg, James ou encore Ibsen oubliés sur le palmarès, ou mis hors de combat par le secrétaire perpétuel. Par exemple Tolstoï est hors-jeu du fait de son «hostilité envers la culture». L'interprétation du mot clé «idéalisme» du testament est interprété par le secrétaire Wirsén comme suit: «une véritable noblesse, qui ne tienne pas seulement à la façon de présenter les choses mais qui marque également sa façon de les concevoir ainsi que sa philosophie» (Espmark, 1986, p. 244). Durant cette période, le prix Nobel littéraire «n'est pas avant tout un prix littéraire, les qualités purement artistiques sont mises en balance avec l'appui apporté à l'humanité dans ses efforts pour atteindre les idéaux». Comme le mentionne le tableau 2, les lauréats du prix durant la Première Guerre mondiale seront ceux qui auront opté pour une «politique de neutralité», «les petites nations qui se tiennent à l'écart des blocs» seront favorisées, en d'autres termes les littératures nordiques, à l'exception notable du nobéliste français, Romain Rolland qui se distingue de toute façon par ses articles anti-guerre (1914-1918) et dans ses œuvres par une attitude défensive de l'indépendance des colonies françaises.

Tableau 2 : Lauréats du prix Nobel de littérature pendant la Première Guerre mondiale

\begin{tabular}{|l|l|l|}
\hline Année & Pays & Nom - Prénom \\
\hline 1914 & Non décerné & - \\
\hline 1915 & France & Romain Rolland \\
\hline 1916 & Suède & Verner von Heidenstam \\
\hline 1917 & Danemark & Karl Adolph Gjellerup, Henrik Pontoppidan \\
\hline 1918 & Non décerné & - \\
\hline 1919 & Suisse & Carl Spitteler \\
\hline
\end{tabular}

À l'heure actuelle, plusieurs spécialistes regrettent encore les oublis majeurs sur le palmarès qui représentent «les monuments de la littérature universelle» (Espmark, 1986, p. 152) tel que Virginia Woolf, Marcel Proust, James Joyce, Robert Musil, Witold Gombrowicz. Une autre critique qui parait encore plus sévère est le fait que certains auteurs ayant reçu cette récompense soient vus comme «indignes du prix». Nous pourrons citer parmi ces auteurs, le premier lauréat de 1901 qui est Sully Prudhomme suivi de Paul Heyse (1910), Rudolf Christoph Eucken (1908), José de Echegaray (1904). Le comité regrette amèrement d'avoir attribué cette gloire à Bjørnstjerne Bjørnson au lieu d'Ibsen. Une autre interprétation du testament de Nobel voit le jour avec l'arrivée du nouveau secrétaire perpétuel Österling en 1941. «Ce qu'il exige d'une œuvre littéraire, pour qu'elle puisse être prise en considération, c'est une philosophie mûrement réfléchie et de l'humanité». Certains auteurs, bien qu'ils soient récompensés, ils le refusent. Comme Boris Pasternak qui se doit de refuser le prix qui lui a été décerné en 1958 pour des raisons politiques, par crainte d'être 
exilé de son pays. Il tente de s'épargner des sanctions en déclinant ce prix, dans le but d'éviter de déclencher à nouveau la colère des autorités soviétiques. Quant à Jean-Paul Sartre, lui refuse de bon cœur la récompense qui lui a été accordée pour de simples raisons personnelles et objectives. Notamment il dira dans sa lettre qu'il écrit pour le secrétaire que: «L'écrivain doit donc refuser de se laisser transformer en une institution même si cela a lieu sous les formes les plus honorables comme c'est le cas» en ajoutant que

Le seul combat actuellement possible sur le front de la culture est celui pour la coexistence pacifique des deux cultures, celles de l'Est et celle de l'Ouest. Je ne veux pas dire qu'il faut qu'on s'embrasse, je sais bien que la confrontation entre ces deux cultures doit nécessairement prendre la forme d'un conflit, mais elle doit avoir lieu entre les hommes et entre les cultures, sans interventions des institutions. [...] Mes sympathies vont indéniablement au socialisme et à ce qu'on appelle le bloc de l'est, mais je suis né et j'ai été élevé dans une famille bourgeoise. [...] J'espère cependant bien entendu que « le meilleur gagne», c'està-dire le socialisme.

Le refus du prix dans un passé récent, face à la pression de la presse et de la droite allemande, qui demandent à ce que Günter Grass rende le prix qu'il a reçu en 1999, puisqu'il s'est engagé volontairement au sein de la Waffen-SS en 1944, pousse le secrétaire perpétuel Peter Englund à intervenir et à déclarer qu' «il n'y a pas, et il n'y aura pas, de discussions à l'Académie suédoise pour lui retirer son prix» 4 .

En rejetant un simple coup d'œil sur la liste des lauréats on constate que le roman ainsi que les autres genres narratif sont les plus primés et qu'il n'y a simplement que treize femmes qui ont reçu le prix Nobel de la littérature depuis son histoire (cf. le tableau ci-dessous).

Tableau 3: Liste des lauréates ayant obtenu le prix Nobel de littérature

\begin{tabular}{|l|l|}
\hline Nom des lauréates & Année \\
\hline Selma Lagerlöf (1858-1940) & 1909 \\
\hline Grazia Deledda (1871-1936) & 1926 \\
\hline Sigrid Undset (1882-1949) & 1928 \\
\hline Pearl Buck (1892-1973) & 1938 \\
\hline Gabriela Mistral (1859-1957) & 1945 \\
\hline Nelly Sachs (1891-1970) & 1966 \\
\hline Wislawa Szymborska (1923-2012) & 1966 \\
\hline Nadine Gordimer (1923-2014) & 1991 \\
\hline
\end{tabular}

\footnotetext{
${ }^{4}$ Article du journal Le Point, 10 avril 2012, «L'Académie suédoise exclut de retirer le Nobel à Günter Grass ». Lire en ligne http://www.lepoint.fr/culture/l-academie-suedoise-exclut-de-retirerle-nobel-a-gunter-grass-10-04-2012-1450090_3.php, consulté le 2 décembre 2014.
} 


\begin{tabular}{|l|l|}
\hline Toni Morrison $(1931-\ldots)$ & 1993 \\
\hline Elfriede Jelinek $(1946-\ldots)$ & 2004 \\
\hline Doris May Tayler-Lessing $(1919-2013)$ & 2007 \\
\hline Herta Müller $(1953-\ldots)$ & 2009 \\
\hline Alice Munro $(1931-\ldots)$ & 2013 \\
\hline
\end{tabular}

Il est donc intéressant de constater que le prix Nobel de littérature est essentiellement masculin, Ce nombre inférieur de femmes peut-être regrettable et peut peut-être s'expliquer par des raisons sexistes, politiques, voire surtout sociales. À l'époque il y avait moins d'écrivaines célèbres que d'écrivains dû au rôle réducteur des femmes imposé par la société, la femme a longtemps vu son rôle minimisé voire limité dans le monde universitaire / académique pour des raisons avant tout sociales (héritage des sociétés patriarcales).

En observant le graphique 1 qui met en évidence la réception du prix Nobel par pays, on constate que l'Hexagone représente le pays ayant le plus reçu ce prix, avec quinze lauréats, suivi des Etats-Unis, puis du Royaume Uni, Allemagne etc... La Suède se trouvera une place qu'au cinquième rang avec huit lauréats. Lors d'une entrevue accordée à l'agence Associated Press, Horace Engdahl (secrétaire perpétuel de l'académie suédoise entre 1999 - 2009), partage l'idée qu'il ne faut pas s'étonner si la «plupart des lauréats du prix sont européens» et qu'il «existe bien sûr des auteurs forts dans toutes les grandes cultures, mais qu'on ne peut nier le fait que l'Europe est toujours le centre du monde littéraire» et non pas les Etats-Unis. De plus c'est en 1913 que nous sortons, pour la première fois, du cadre européen avec le prix accordé à Rabindranath Tagore (1861 - 1941), un indien qui a tout de même reçu une éducation anglaise.

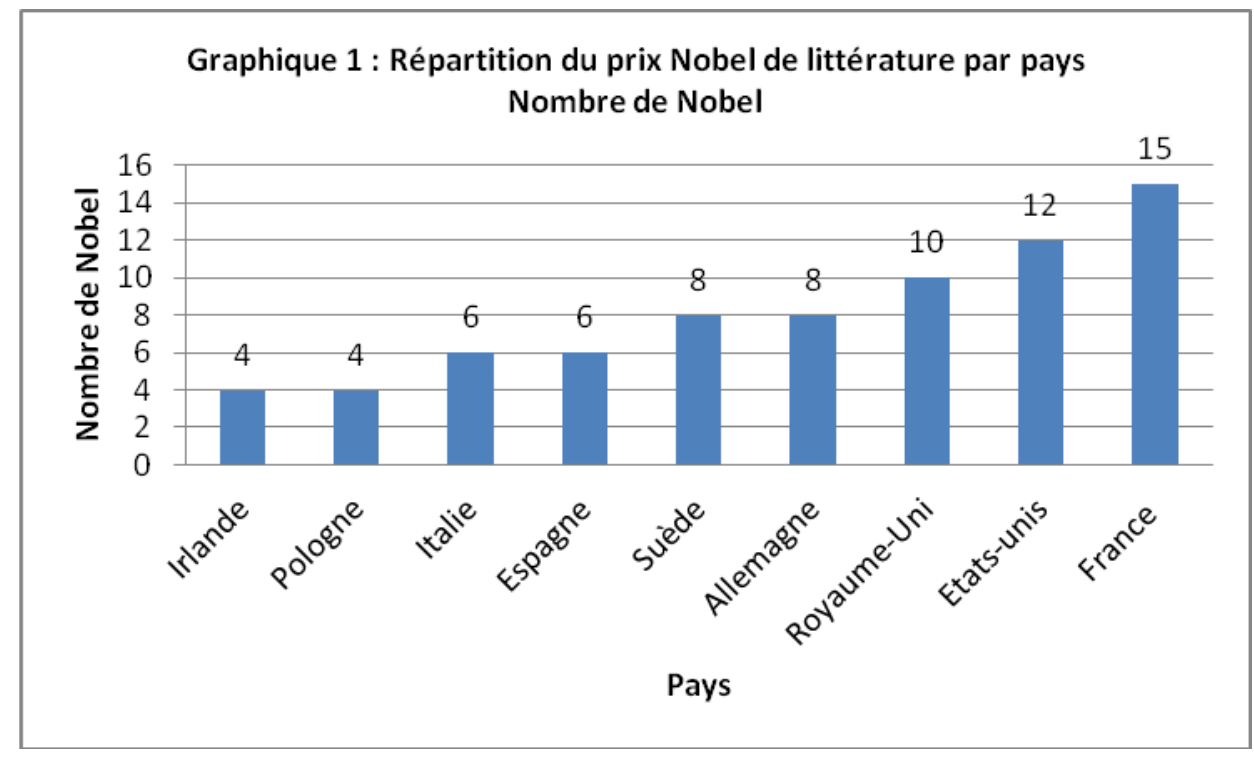


Le graphique 2 met l'accent sur l'importance de la langue choisi par l'écrivain, ainsi que du rôle que joue la traduction pour atteindre le comité de Nobel, Ainsi bien que Alfred Nobel voulait donner une «portée mondiale» aux prix qui sont décernés en son nom, la langue de l'écriture, dans laquelle s'exprime le romancier, joue un rôle primordial. Il est à constater que bien que l'anglais soit la langue la plus primée, considérée comme la langue véhiculaire, dans le monde entier, il serait intéressant de comprendre la raison qui fait que la France est le pays qui soit le plus récompensé, sachant que ses auteurs œuvrent dans leurs langues maternelles. Est-ce parce que la langue française est toujours et encore utilisée comme langue de prestige par l'aristocratie européenne?

Cependant comment expliquer que la langue la plus couronnée est l'anglais alors même que la France détient le record avec ses œuvres écrites en français ? Puis est-ce les langues «inhabituelles» peuvent-elles songer à l'obtention de ce prestigieux prix.

L'écrivain chinois Tsu- Yü Hwang s'interroge sur la question de savoir si un jour un écrivain chinois, écrivant dans sa langue d'origine puisse avoir les mêmes chances qu'un écrivain œuvrant dans une des langues occidentales. En ce sens, les dix-huit membres du comité, ne peuvent évidemment pas avoir accès aux œuvres dans leurs langues d'origines. Or pour cela ils engagent des personnes expertes dans les langues dans lesquelles les œuvres ont été écrites. D'ailleurs l'Académie considère que le temps est enfin venu de couronner un écrivain qui nous transmet sa culture dans sa langue, qui leur est étrangère : elle est tout à fait consciente néanmoins qu'elle ne peut «déléguer sa responsabilité à des experts et doit finir par prendre position elle-même» $\rangle^{5}$.

Ainsi telle est la question qui surgit «est-ce que la langue d'origine des auteurs a un effet sur l'obtention du prix?» (Küçüksüleymanoglu, 2014, p. 10). Il va de soi que depuis que la traduction a gagné de l'importance (à partir des années 1960) et, est devenue vitale pour la diffusion des œuvres sur le terrain de la littérature mondiale, les auteurs hors des frontières occidentales ont eu l'opportunité d'être parmi les lauréats.

\footnotetext{
${ }^{5}$ Testament d'Alfred Nobel.
} 


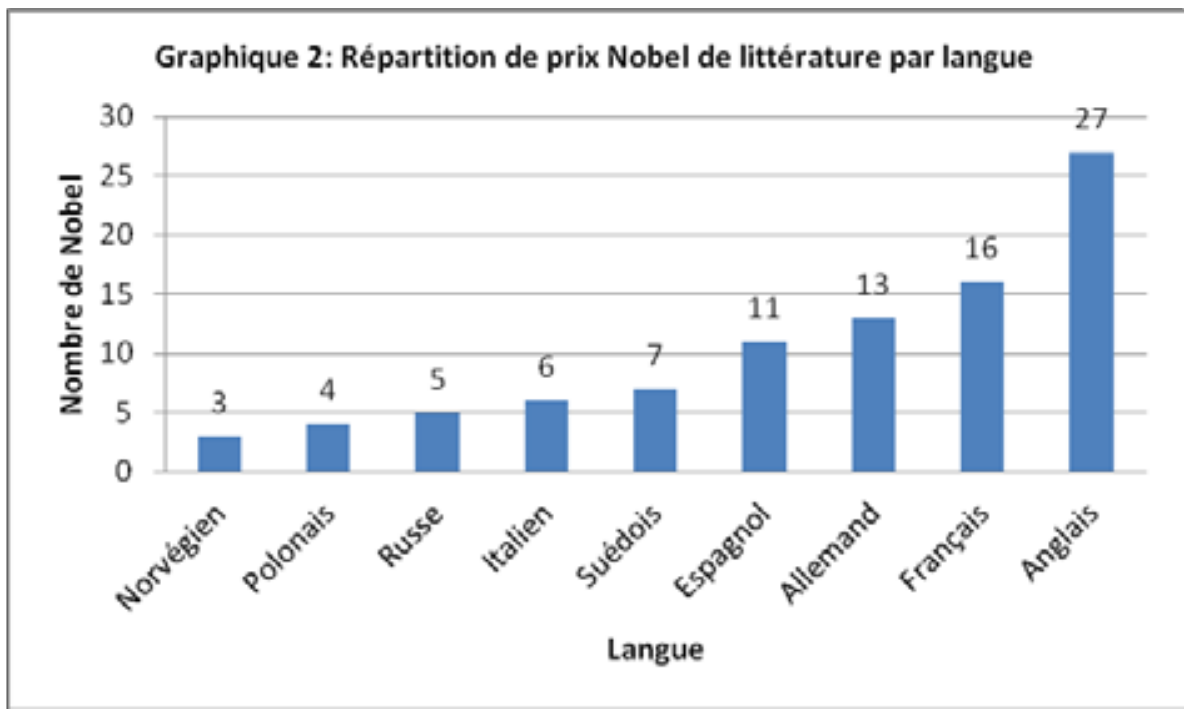

\section{En guise de conclusion}

Avec toutes les questions que le prix Nobel engendre, il fera toujours parler de lui. Toutes ses attributions donneront lieu à des controverses. Il faut cependant ne pas négliger que c'est une question d' «épreuve internationale». Par conséquent il ne touche pas simplement que le monde littéraire, mais toutes les nations qui veulent apporter leurs «petites» pierres à l'édifice culturel mondial, étant donné que les œuvres littéraires servent à devenir des ponts entre les cultures. C'est donc, au tribunal littéraire de juger si tels ou tels auteurs avec leurs talents littéraires ont su «rendre à l'humanité les plus grands services». Son caractère international lui donne non seulement un rayonnement international au niveau mondial étant donné qu'elle occupe une place à part entière dans la communication culturelle mondiale mais aussi une implication politique qui a, depuis les premières attributions du prix, été un sujet à controverse du fait de son caractère irrémédiable. Quand bien même le prix Nobel, âgé de 103 ans, devrait s'écarter de toutes interprétations d'ordre politique et éviter de devenir un problème politique national, il est intéressant de voir que beaucoup de prix ont pris la forme de prix politique. Le comité Nobel a une tendance gauchiste, au sens européen, et c'est peut-être la raison pour laquelle un des très grands écrivains du XX ${ }^{\text {ème }}$ siècle Jorge Luis Borges (18991986), ne se voit pas décerné ce prestigieux et remarquable prix. Notamment un des prix qui a longtemps été discuté pour des raisons politiques est celui de Frans Eemil Sillanpää (1888 - 1964) en 1939 qui était vu par la Finlande comme un ennemi. En outre le prix attribué à l'historien Churchill est aussi considéré comme un prix à caractère politique étant donné qu'il était toujours Premier ministre. Et c'est d'ailleurs ce prix qui a certainement dû mettre en péril l'intégrité politique de l'Académie. En outre les derniers choix de l'Académie n'ont pas fini de semer les doutes du grand public ainsi que des presses. La prise de position contre la guerre d'Irak aurait-il fait d'Harold Pinter le lauréat de 
2005?, tout comme la déclaration qu' «un million d'arménien et 300000 kurdes ont été tués, et personne n'ose le dire» aurait-il fait d'Orhan Pamuk, le lauréat de 2006? Il est à noter que le prix décerné à Orhan Pamuk coïncide le même jour où le parlement français a adopté «une résolution faisant de la négation du 'génocide arménien' un crime» a attisé la tristesse, la colère du public turc et a endommagé l'image de Pamuk auprès de ce dernier.

Toutefois ces contradictions nous poussent à réfléchir sur le sujet, surtout lorsqu'on sait que les membres de l'académie suédoise sont élus à vie. En ce sens peut-on remettre en cause les choix de l'académie suédoise? En général dans tous les pays démocratique où la démocratie impose un renouvellement de ceux qui ont le pouvoir, le comité du prix Nobel devrait-il pas être mandaté? Ce qui souligne la faille de cette dernière, puisque nous vivons dans un monde voué à l'évolution, au changement, dans lequel les œuvres littéraires ont aussi besoin d'être évaluées selon leur époque par des personnes ayant des goûts littéraires différents.

Un autre problème est que la mondialisation qui détruit les frontières nationales et impose une uniformisation des goûts dans tous les domaines, pousse à l'utilisation de l'anglais comme la langue universelle de communication. Les maisons d'édition qui favorisent la publication en anglais, incitent les écrivains à s'exprimer en cette langue afin d'atteindre un maximum de lecteurs qui influencera aussi le chiffre de vente. Dans un tel monde monopolisé au sens culturel et linguistique, le prix Nobel de littérature futurs permettra - $t$-il de faire face à la minorisation culturelle, littéraire et linguistique?

\section{BIBLIOGRAPHIE}

Espmark, K. (1986). Le prix Nobel, histoire d'une consécration littéraire. Paris: Balland.

Gökalp, E. (2010). Pride and anger: Orhan Pamuk's Nobel Prize and discourses of nationalism. Anadolu Üniversitesi Sosyal Bilimler Dergisi, 10(3), 171-190.

Küçüksüleymanoĝlu, Ü. (2014). Nobel Edebiyat Ödülü’nün Yapısı ve Dünya Edebiyatındaki İ̧slevi. (Yayınlanmamış Yüksek Lisans Tezi). İstanbul: İstanbul Bilgi Üniversitesi Sosyal Bilimler Enstitüsü.

Nobel, A-B. (27.11.1895), La volonté d'Alfred Nobel. Disponible en ligne: http://www.nobelpreis.org/francais/\#volonte.

Soyak A. (2003). Nobel İktisat Ödülleri Üzerine Bir Yorum. Finans \& Politik ve Ekonomik Yorumlar, (256), 74-87.

Tétu de Labsade, F. (1997). Littérature et dialogue interculturel : culture française d'Amérique. Québec: Presses de l'Université Laval.

Vural, İ. (2008). Nasıl bir medya: Uzlaşan mı çatışan mı? Orhan Pamuk ve Nobel Ödülü üzerine bir değerlendirme. T.İlter, N. Kara, M. Atabey, Y. Arslan and M. Orun (Eds.). Communication in Peace/ Conflict in Communication 
(pp.143-152). North Cyprus, Famagusta: Eastern Mediterranean University Press.

\title{
WHY HAD NOBEL PRIZE BEEN CRITICIZED?
}

\begin{abstract}
The Nobel Prizes, like the Oscars and Grammys in the film and music industry, are one of the most prestigious awards in the world, and they are eagerly awaited by the general public. The prizes given by the Royal Academy of Sweden, without making a distinction of language, religion and nationality, in the fields of Physics, Chemistry, Literature, Medicine and Economics have long been controversial as to whom, for what reason and how they are awarded; and the laureates occupy the news headlines for a long time. Alfred Nobel (1833-1896), who invented dynamite, and supposedly committed a crime against humanity, had announced in his will that he bequeathed his fortune to those who have contributed to humanity, and that has long caused a perpetual debate on the basis of the prizes. Another issue that is current at the awards is whether the choices are objective. Are these prizes, distributed by the Swedish Academy, really reliable? Are the laureates promised to be awarded earlier? In this work, we try to answer all these questions and more (which country has the most received the Nobel Prize? What is the most advocated language? To receive the Nobel Prize writers should they be engaged? The writers were they as likely as writers?) precisely we study the issue through the Nobel Prize for Literature, which has always been at the heart of discussions on the matter.
\end{abstract}

Keywords: The Nobel Prize for Literature, Politics, the Royal Academy of Sweden, Authors, Books. 